\title{
Functions of language in the social context
}

\author{
Alyona Korneeva ${ }^{1, *}$, Tatyana Kosacheva ${ }^{2}$, and Oxana Parpura $^{2}$ \\ ${ }^{1}$ Department of Foreign Languages, Economic and Legal Profiles, Altai State University ASU, 68, pr. Sozialisticheskij, 656049, \\ Barnaul, Russia \\ ${ }^{2}$ Department of Foreign Languages, Altai State Agricultural University ASAU, 98, pr. Krasnoarmeyskiy 656049, Barnaul, Russia
}

\begin{abstract}
The article considers the language in use, in the process of interaction and as a sign system used in the process of communication, describes the functions of the language. The language is a system of discrete signs that serve to communicate and express the totality of person's ideas of the surrounding reality, and it is a social phenomenon. The basic functions of a language are interdependent when using the language and are implemented in various degrees. Researchers identify different functions of the language; many classifications depend on its ways of use. Social stipulation of the language is manifested in the expansion of the scope of the functions, the diversity of its forms, functional styles.
\end{abstract}

\section{Introduction}

Culture is an important mechanism of human interaction, which helps people to live in their environment, to preserve the unity and integrity of the community, while interacting with other nations. The knowledge formulated in certain concepts and representations and recorded in the language is one of the important elements of culture, the language being a cognitive, sign, symbolic element of culture. Language is an objective form of accumulation, storage and transfer of human experience. Language as an element of culture is a system of signs and symbols possessed with a certain meaning.

Language as a sign mechanism of communication, a system of discrete signs that serve to communicate and are able to express the totality of a person's potion of the world as a social phenomenon. The social stipulation is expressed in the social functions of the language as a means of people communication. The word conveys some information, reinforces accumulated knowledge, delivers emotional pleasure or displeasure, orders or expresses a desire, etc. The affiliation of the language to social phenomena is manifested in the fact that the language is a keeper of information about the past of mankind, about the history of peoples, nations. The language reflects and consolidates the realities, abstract concepts developed by the people's historical experience, owing their existence to the specific conditions of the working, social and cultural life of the certain people. The national-cultural function of the language is reflected in the language itself. The specificity of the understanding of the same concept or phenomenon by the certain people is largely reflected in the peculiarities of the name, in the specific semantic changes, in the nature of the language reflection of surrounding reality. Language as an instrument of knowledge makes it possible to get new information about the reality that surrounds us, producing only theoretical activities mediated by the language, and not referring directly to practical activities. In the personality formation and education, the language in human society performs the following functions: the communicative and cognitive functions and the influence function.

\section{Materials and methods}

The main methods of our research are general scientific methods - analysis, in particular, analysis of the literature on the research topic, synthesis, historical methods, comparison, abstraction, concretism, generalization.

\section{Results}

Language as the most important means of human communication, as a social phenomenon performs a number of functions in the life of society.

The word «function» (from the Latin functio «fulfilment») has several meanings. In everyday use, it means such things as: meaning, purpose, role; duty, terms of reference; work, activity; a certain phenomenon, depending on another, the main phenomenon and serving as a form of its manifestation, implementation. In various meanings this word is used as a scientific term, i.e. has a variety of special meanings. As a linguistic concept, it is also multivalued. According to some linguists, this term has several meanings in the science of language [1].

The compound linguistic term «function of language» or «language function» denotes the purpose,

* Corresponding author: korneevaalyona1@rambler.ru 
intended purpose, or «predestination, potential orientation of the language system to meet the needs of communication and the needs of mental activity» $[2, p$. 67]. Following V.A. Avrorin, the concept of the language function can be defined as «the practical manifestation of the essence of language, the realization of its purpose in the system of social phenomena, the specific action of language due to its very nature, without which language cannot exist, as there is no matter without movement» $[3$, p. 34].

Considering the language functions, many researchers analyze the functions of the language as a universal phenomenon, i.e. the functions inherent in different languages.

The theory of the language functions was created by $\mathrm{K}$. Buhler, who defined the functions of the language as follows: an expression function, or an expressive function, when the speaker's state is expressed; the function of call, appealing to the hearer, or the appellate function and the function of representation or the representative function [4].

At present it is necessary to single out different aspects of the functional approach to language phenomena, therefore the problem of functions is more extensive. M. Halliday relates the functions of the language to the formal apparatus of their expression; at the same time, the situational context has a significant influence on the statements formation and on the functioning of the language system. The author identifies three main functions of the language:

1) ideational or the experiential function;

2) interpersonal or social role function;

3) textual or discursive function determines the connection of the language with the situation in which it is used and allows the user to create the text, that is, connected, situationally determined segments of discourse.

These functions are used to determine the semantic potency, consisting of a huge number of choices when using language. The system of choices is the grammar of this language. In the statement all functions are implemented simultaneously, but each of the grammatical contrasts is based on one of them. Modality is associated with the interpersonal function; procedurality - with the conceptual function, the textual one turns the sentence into a statement [5].

Defining the functions of the language, R.O. Jacobson considered the model of speech communication consisting of six elements: an addresser, an addressee, context, message, contact, code. The special function of the language corresponds to each of the elements of speech communication. The «function of the language» is understood there as «the arrangement or the appointment of the message itself in relation to other factors of verbal communication». The message performs several functions, and the verbal structure of the message depends on the prevailing function. R.O. Jacobson identifies the following functions in the communicative act:

The emotive function is focused on the speaker, his emotional state. It is associated with the desire to impress or cause certain emotions of the recipient.
Conative function is focused on the addressee, expresses a direct impact on the interlocutor. Reference or communicative function correlates with the subject in question. This function is related to the context, directed to the described situation, its essence. The metalinguistic function is related to the language code and is directed to the interpretation of language units. The phatic function realizes the goals of contact maintaining, its purpose is to determine whether to continue or interrupt the communication. The poetic function is focused on the form, not on the content, on the aesthetic aspect of the statement [6].

This model describes communicative processes, takes into account not only the language itself, but also the actively involved user.

A.A. Leontiev identifies the following functions: communicative, tools of intellectual activity in general, mastering the social and historical experience of mankind, national-cultural, tools of cognition. The function is an essential attribute of the language, the form of its existence. What is present in every speech act is a function of the language [7].

\section{Discussion}

Language researchers have different opinions about the number and nature of functions, but the basic ones are communicative and cognitive.

The cognitive function of a language (from the Latin cognition - «knowledge, cognition») is connected with the fact that the human consciousness is realized or fixed in the signs of the language. The cognitive (thinking) function is a means of obtaining new knowledge of reality, this language function connects it with the human mental activity, the structure and dynamics of thought materialize in language units. With the help of language the thought is materialized; it acquires a concrete and tangible form that can be perceived by the sense organs of another person. Thanks to language the thought is formed, the language participates in the process of the thought emergence, since language is not simply the materially real form of the thought existence, the thought «clothing».

The word as static thought, which has assumed the objective form of existence, simultaneously acts as a dynamic form of thought, unfolding within the time framework; therefore it participates in the generation of thinking. In this mutual generation, language performs the function of thought formation - the thoughtforming function. Its practical implementation occurs in speech, when verbality is necessary, that as autodialogue or external dialogue. The words not only express our thoughts, but thoughts themselves exist in the form of words, verbal formulations. Any images and concepts of consciousness are realized by man and others only when they are exposed in a linguistic form, therefore the connection between language and thinking is inseparable. A concrete thought, like other thoughts, is a subjective process of reflection by the consciousness of a certain "piece" of objective reality, the ability of consciousness to reflect reality and its ability not only to 
detect a real universal connection in things, properties and relations, but also to create a surreal connection between these realities. The creation of a surreal connection is determined by the need to replenish the limited possibilities of consciousness, which perceives not the essence of things, properties and relationships, but only their phenomena, by the necessity caused by the social-production being of a person. This particular thought is connected with the social development of a person conditioned by him and therefore must be expressed, spoken to other members of society. So you need a way to design it, a way to «transfer» the ideal (thought) from one material substance (brain) to another material substance. The material shell of the language in its oral and written forms, is one of the material substances.

The cognitive function of the language allows you not only to record the results of mental activity and to use them in communication. It assists to know the world. A person's thinking develops in categories of language: realizing new concepts, things and phenomena the person names them. The cognitive process is the process of acquiring true knowledge of the objective world in the course of social and practical activities of society [8].

The objective world is reproduced by thinking, the results of this process are recorded in language structures. Summarizing the mass of concrete phenomena, a person, distracting from their random signs and highlighting the essential ones, feels the need to consolidate the obtained knowledge in the word, so the name appears. The nominative language function is associated with the ability of signs to mark things. The world is known and explored only when it is named. The name allows you to fix everything already cognizable. Without a name, any known fact of reality remains in consciousness as accidental. Calling words, a person creates his own picture of the world. Words are the names of objects, their mental image. In the process of thinking the person operates with the names of objects, which often replaces mental images and increases the speed and efficiency of thinking.

With the help of language, a person expresses a thought - an ideal image of an external person. Thanks to language, thought is expressed as a subjective image of the objective world and in this way language performs the function of thoughts expression — an expressive function. The processes of formation and expression of thoughts through language occur simultaneously, since they cannot be carried out separately. At the same time, these are different processes: the formation of the thought is its transfer to a materially-ideal "thing," transformation into language forms; the expression of a thought is its other-being re-creation in space and time outside the human brain. The product of mental activity is knowledge.

«Knowledge is a result of the reality cognition verified by social and historical practice and logically proved, the adequate reality reflection in human consciousness in the form of representations, concepts, judgments, theories» $[9$, p. 45]. Knowledge, as a rule, is reproduced in the language form. Every word, statement, language category means the essential layer of knowledge. The linguistic form is a reflection of cognitive structures, i.e. structures of human cognition and consciousness [10].

Language is a means of expressing of the inner person's world, it not only transfers an informational message, but contains the attitude to the statement, in which the speaker manifests his feelings, experiences, emotions. With the expressive function the language serves as a means of expressing the inner state of the speaker, expresses the speaker's attitude to the information content, to the interlocutor, to the communicative situation. Language expresses not only thoughts, but also human emotions. A person spontaneously or consciously conveys his mental attitude to what is happening.

In the sphere of communication, the leading function of language is its communicative function, it allows one individual - the speaker - to express his thoughts, and the other - the perceiver - to understand them, react, take note, change their behavior. The communicative function of the language is realized on the basis that the language itself is a system of signs. To implement this function, the language was formed as a system of signs, combined with each other according to certain rules, due to them human thought is materially expressed and perceived. Language is a communicative system, on the basis of which speech statements are made. As for the content, the language allows you to express a great number of thoughts and ideas using each time the minimum set of words and grammar means. This function mediates individual content through individual speech and turns into a social phenomenon, emphasizing the unity of the communicative, gnoseological and social functions of the language.

The gnoseological function is that language is a peculiar system of reference points in the perception of the world, an instrument for information processing. When we recognize an object, we name it as a word, and the word itself is related to other words: in this way the object is connected with other objects through the language. Besides, the meanings of words themselves contain a list of characteristic features of an object named with the word, which makes it possible to manipulate the concepts about objects with the help of words and even to conduct experiments that are unthinkable for real objects. Language in terms of vocabulary and word formation is one of the powerful tools of thinking.

Sensory perception is a simple way of knowing the outside world, but not all objects, their signs and properties are perceived and understood by the senses. Abstract concepts, such as space, movement, speed, etc., are not available to sensory perception. The sense organs give a superficial idea of specific objects. The deep and comprehensive cognition of the world around us is possible only with the help of language.

The language participation in the knowledge of reality is manifested in the process of thinking, in the formation of concepts and judgments that are expressed in words and sentences. Without the language participation and language means the scientific, research activity of people, which constantly enriches us with 
new information about the world and environment, is simply impossible.

In the process of learning communication of people plays an important role in order to exchange information and experience. Such an exchange is possible not only through direct oral communication, but also when reading books, newspapers, magazines, while listening to radio broadcasts, watching television, films, theater plays, etc. This is possible with the participation of the language.

The source of human knowledge is not only specific language units, but also certain language categories, in particular grammatical ones. For example, a noun as a part of speech means an object (in a broad sense), an adjective is an object feature, a numeral is a number, a number of objects, a verb is an action, a process. The same can be said about the lexical and grammatical categories of nouns, adjectives and other parts of speech, categories of number, gender, animation, degree of comparison, time, mood, etc. [11].

Communication is the means of implementing mental content, which is a cognitive prerequisite that forms an informative basis. A message is a complex unit in which at least two categories exist: about what information is reported and what is reported. This universal construction of any communication is the first condition for the exchange of thoughts in the human team, and it is the first condition that qualifies position and semantics of a language unit as a moment of a certain chain, context.

All the information produced by mankind exists in a language form. Each piece of this information can be pronounced and perceived by both contemporaries and descendants. This is the accumulative function of the language through which the humanity accumulates and transmits information, both in modern times and in historical perspective. The accumulative function ensures the continuity of knowledge and the existence of a person in history.

Any language, accumulating the experience of national life in all its fullness and diversity, is also its real consciousness. Each new generation, each representative of a specific ethnos, learning language it joins the collective experience, collective knowledge about the surrounding reality, generally accepted standards of behavior, estimations rejected or accepted by people, social values. Language affects the experience of a particular individual, his behavior, culture.

Language is the storehouse of the ethnos culture, the reservoir of knowledge about the world. The language embraces the entire cognitive experience of the people, its moral and ethical, social and aesthetic, artistic and educational ideals. The language keeps the history of the movement of the people along the path of civilization, it reflects the people character, their sympathies and antipathies, and relations with neighboring peoples. The language absorbed all the details of the evaluative attitude to reality, its perception and reflection.

The accumulative function of the language is based on the most important purpose of the language - to collect and store information, evidence of human cultural activities. The accumulative function of the language ensures the scientific, technical and cultural progress of mankind. With its help the times and generations are connected, the acquired knowledge and experience is spread among people, becomes the property of different nations, is passed from generation to generation, which ensures the accumulation and continuous enrichment of experience and knowledge, the development of science, technology, etc.

Representatives of hermeneutics (the art of texts interpreting), consider language as a way of human existence, they pay attention to the motivating and evaluative functions of the language. From these positions, the emotional, aesthetic, ethical impact of the word, its potential convincing power, suggestion and evaluation, motivation and control reveal their priority significance for human activity.

\section{Conclusion}

Different types of human social activities are served by different functional language varieties, dialogue being the most common form of social and speech influence. Communication in all its forms, types and genres allows a person to get ready social experience, sensible and systematized by previous generations. The role of dialogue in the life of mankind is extremely large. Dialogue and the communication act as the most important characteristics of person cultural creativity. Axiological worlds in the process of communicative interaction can reflect both individual and group or social values. Dialogue can be considered as a form of creativity, a form of person development, his culture and social world.

In virtue of this analysis, it follows that the language, being the most important means of communication and unification of people, regulates their interpersonal and social interaction, coordinates their practical activities, participates in the formation of ideological systems and national images of the world. It also ensures the accumulation and storage of information, classifies and fixes concepts, forms the consciousness and selfconsciousness of man. It should be noted that the natural difficulty of classifying and discussing the functions of the language is that the ways of language usage are so diverse and numerous that no classification can be considered complete and satisfactory. It is quite obvious that various functions of the language are combined, intertwined, give rise to variants and varieties and new socially significant functions of the language are born. With the help of language, a person realizes the role of his people in the past and present, joins the cultural heritage, the modern processes of the spiritual development of society and nation.

\section{References}

1. R. Jakobson, Form und Sinn. Sprachwissenschaftliche Betrachtungen (Wilhelm Fink Verlag, Munich, 177, 1974) 
2. L.A. Kiseleva, Communicative language functions and semantic structure of word meaning (Problems of semantics, M., 1974)

3. V.A. Avrorin, Problems of studying of language functional side (Revisiting the subject of sociolinguistics, L., 276, 1975)

4. K. Buhler, Theory of Language: The Representative Function of the Language (Moscow: Progress, 504, 1993)

5. M.A.K. Halliday, An Introduction to Functional Grammar (London: Arnold, 387, 1985)

6. R.O. Jacobson Works on Poetics (Moscow: Progress, 464, 1987)

7. A.A. Leontiev, Language. Speech. Speech activity (M., Enlightenment, 214, 1969)
8. I.G. Zhogova, E.V. Kuzina, Language and culture, Development of critical thinking in the context of vocational-oriented teaching of high school students, $\mathbf{3 8}$, 227 - 239 (2017)

9. E.S. Kubryakova, Questions of linguistics, The initial stages of the cognitivism formation: linguistics psychology - cognitive science, 4, 34-46 (1994)

10. F.R. Imamutdinova, Bulletin of KSU named after O.N. Nekrasov, The implementation of the communicative and cognitive functions of the language in speech activity, 1, $78-80$ (2010)

11. B. Götz, Sprechakte und Sprachfunktionen: Untersuchungen zur Handlungsstruktur der Sprache und ihren Grenzen (Tübingen: Niemeyer, 266, 1980). 